\title{
Análisis de tendencias en movilidad en el Gran Valparaíso. El caso de la movilidad laboral ${ }^{1}$
}

\author{
Marcela Soto Caro ${ }^{2}$ y Luis Agustín Álvarez Aránguiz 3
}

\begin{abstract}
RESUMEN
Una de las transformaciones urbanas fundamentales a nivel sudamericano son los procesos de tránsito entre conurbaciones y áreas metropolitanas, donde la promoción de la movilidad por una serie de factores descritos en este artículo, genera y promueve dinámicas territoriales de nuevo tipo. Podemos señalar que hoy nos enfrentamos a mayores movilidades por trabajo y cada vez más complejas desde la perspectiva de los patrones históricos de los "lugares de trabajo" versus "lugares dormitorio", la realidad contemporánea los superpone mezclándolos, produciendo asimetrías entre las distintas unidades territoriales medidas. Estas características territoriales tanto de tipo sociodemográficas, económicas como propiamente físicas (densidades, tipologías edificatorias, habilitación de redes de infraestructura, etc.) explican diferencias en los desplazamientos al trabajo que se generan en el caso del Valparaíso Metropolitano.
\end{abstract}

Palabras clave: Movilidad laboral, áreas metropolitanas, infraestructuras.

\begin{abstract}
One of the fundamental urban transformations at South American level are the processes of transition between conurbations and metropolitan areas, where the promotion of mobility for a number of factors described in this article, produces and promotes new type of territorial dynamics. We can point out that today we face greater mobility for work and increasingly complex from the perspective of historical patterns of "workplace" versus "dormitory places", the contemporary reality mix them, producing asymmetries between the different territorial units. These territorial characteristics of socio-demographic, economic and physical proper (densities, building types, enabling network infrastructure, etc.) explain differences in commuting generated in the case of the metropolitan Valparaiso.
\end{abstract}

Key words: Labor mobility, metropolitan areas, infrastructures.

1 Artículo recibido el 6 de febrero de 2011, aceptado el 5 de marzo de 2012 y corregido el 23 de abril de 2012 .
2 Universidad Técnica Federico Santa María (Chile). E-mail: marcela.soto@usm.cl

3 Universidad Técnica Federico Santa María (Chile). E-mail: luis.alvarez@usm.cl 
Las tendencias respecto de lo urbano y lo laboral en la metropolización se nos presenta integrando más las áreas urbanas y la población circunscritas a ellas, favoreciendo los servicios, y respecto de lo laboral productivo, queda sometido a un fenómeno de dispersión en el territorio, lo que se ha denominado proceso postfordista (Castells, 1991), como un efecto contrario a la concentración urbana que generaba la economía urbana industrial. En este contexto, una de las condiciones dominante para tener acceso al mercado de trabajo, es la movilidad.

El cambio histórico se desarrolla desde la mutación de un proceso productivo basado en el aparataje industrial fordista, cuya función de optimización coloca la unidad de producción y atracción de empleos en el centro de gravedad del territorio configurado por los puntos de localización del empleo, las materias primas y el mercado consumidor. El efecto territorial -en la medida que las industrias incorporan en sus funciones de producción la localización de esos tres puntos- es la concentración alrededor de la unidad de producción de los lugares en donde se vive y se trabaja, de manera que la misma actividad productiva es la que induce y provee la vivienda. La evolución actual de las bases económicas de las áreas metropolitanas hacia actividades terciarias (finanzas, comercio y servicios), ha provocado que las actividades productivas se "desterritorialicen" y se independicen respecto de los centros proveedores de mano de obra, materias primas y los mercados consumidores, cuyo efecto urbano es la pérdida del vínculo territorial directo de la unidad productiva respecto de su entorno urbano (Serratosa, 1997).

Esta cierta "liberación" de las industrias respecto de la localización de sus materias primas, la mano de obra y mercado consumidor que muestran las actividades productivas terciarias, se produce gracias a la movilidad creciente de los factores de producción, los consumidores y los bienes. La "ciudad más afuera" (Hidalgo y Borsdorf, 2005: 189), sería la preferencia territorial de las demandas de nuevas áreas urbanas, configurándose como el elemento central de la nueva organización urbano-territorial. La movilidad, entonces, se ha transformado en un factor sustantivo en la explicación de las dinámicas económicas y las centralidades relacionadas con la ciudad y el territorio contemporáneos. Como concepto, ha abandonado las tradicionales formas de entenderlo vinculado a los procesos migratorios (como las migraciones campociudad propias de la década de los sesenta), y surge como factor importante de evaluación de la calidad de vida de las personas en la ciudad contemporánea. Es común apreciar en los planes estratégicos de las principales ciudades del mundo la movilidad como un capítulo esencial, junto a los relacionados con la calidad ambiental, las centralidades urbanas y la competitividad urbana.

Consultados los habitantes del Gran Valparaíso (820.000 habitantes), a través del censo del año 2002, si estudian o trabajan en esta comuna, el $47 \%$ responde que no, si lo desagregamos por unidades administrativas, los habitantes de Quilpué y Villa Alemana, el porcentaje se eleva al $72 \%$, y en algunas manzanas-condominios de Villa Alemana se eleva por sobre el $95 \%$ de personas que se trasladan fuera del radio de acción de la comuna donde viven. Es lo que se ha denominado a partir de la década de 1980 "ciudades dormitorio", misma fecha que comienza la asimetría en la distribución de población en la unidad de estudio, mientras Valparaíso cede la primacía en cantidad de habitantes (1983) a Viña del Mar, las ciudades del interior se elevan a un $17,8 \%$ y $22 \%$ de crecimiento intercensal, promedio sobre la media nacional de $11 \%$.

Si la media de desplazamiento de hace 10 años dentro del Gran Valparaíso alcanzaba los siete kilómetros, hoy está por sobre los 18 kilómetros promedio de desplazamiento. Una de las causas es la apertura de las rutas interregionales dentro del sistema, desarrollando demandas en distancia muy parecidas a lo que Melvin Webber (1968) ha denominado "comunidades sin proximidad", o "vivir la distancia" (Monclus, 2000), o "flexities" (Soja, 2002). Del mismo modo, la encuesta origen-destino (SECTRA Zona Norte, 19982001), muestra que los bordes de la zona urbana metropolitana del Gran Valparaíso ya se sitúan a una hora de viaje (borde isocrona 60 minutos incorporando Curauma, QuilpuéVilla Alemana, Concón y Reñaca alto) en locomoción colectiva hacia el centro de Valparaíso. 
En las particulares condiciones geográfico-territoriales estos desplazamientos cobran significado respecto de los tiempos de recorridos, relativizados por la fricción de las condiciones topográficas. En este contexto la acción facilitadora de las redes de infraestructura acometidas a partir del cambio en las políticas de obras públicas con la entrada en vigencia de los sistemas de concesiones, constituyen un elemento acelerador de las nuevas accesibilidades construidas en los últimos años en el área metropolitana de Valparaíso y la macrorregión central. Debido a ello aumentan la movilidad de personas y proveedores hacia áreas periurbanas de la ciudad, sumado a un permanente mejoramiento de los equipamientos de apoyo a la movilidad, a saber, actualización del transporte de ferrocarriles (Metroval) dentro del área metropolitana y la licitación del sistema de transporte metropolitano de Valparaíso. Consecuentemente, esto genera una condición de fuerte promoción por localizaciones de vivienda no necesariamente asociadas a los requerimientos laborales, educación, salud y esparcimiento como el "Movimiento Moderno" lo promulgase durante el siglo XX, sino más bien a modos de vida; mayores terrenos residenciales y la incorporación de la vivienda bajo la modalidad preferente de los condominios cerrados (Hidalgo y Borsdorf, 2005; Janoschka, 2002; Rodríguez, 2008), teniendo una misma cercanía al centro en tiempos de desplazamiento. Lo que aumenta es la distancia hacia los lugares de trabajo manteniendo el tiempo como una variable constante debido a todos los cambios en infraestructura (Zahavi, 1980; Palomares, 2008). Finalmente, todo esto tiene un efecto en la extensión de las áreas de influencia de los centros metropolitanos y de los territorios urbanizados en forma creciente.

La sustitución de los vínculos de proximidad, ya anticipados hace tiempo (Webber, 1968) por relaciones de conexión, es consecuente con el papel que han alcanzado las redes de infraestructura (Dupuy, 1987) como organizadoras del territorio, e incluso construyendo los mismos desde su bajo nivel de integración (Allen, 1990). La ruptura de la ciudad continua (Herce, 2009), hizo aparecer sobre el territorio muy variadas formas de ocupación ligadas a las actividades que ya no tenían una dependencia de un núcleo urbano fijo, sino relaciones espaciales con otros espacios ubicados a muy distinta distancia.

Las transformaciones también se suceden como respuesta a las cambiantes necesidades sociales de dar respuestas a los nuevos escenarios productivos descritos anteriormente, alterando sustantivamente los modelos, hasta hace poco abordados como fenómenos migratorios, hoy referidos como modelos sociales de movilidad. Donde estos hoy incrementan el trabajo autónomo y diversificación de los lugares de trabajo

Toda esta dinámica socioespacial se despliega en una topografía accidentada y limitante del crecimiento urbano, donde la posibilidad de realizar desplazamientos alternativos quedan restringidos, derivando en el acceso poco equitativo al suelo y calidad de vida urbana por parte de los sectores de menores recursos (Herce, 2009).

\section{Planteamiento del problema}

En este artículo se indaga la movilidad de la fuerza de trabajo dentro de un contexto definido como "Gran Valparaíso", este proceso de movilidad es el resultado de las interacciones que el espacio metropolitano ha estado generando en el último tiempo, entre ellas, las transformaciones productivas, la generación de nuevos servicios y las transformaciones infraestructurales.

Si bien el área metropolitana de Valparaíso se desarrolló como un área conurbada hasta los albores de la década de 1980, sistemáticamente se incrementó un mayor nivel de integración de servicios y roles en una economía con menos participación estatal en las decisiones territoriales y por tanto reorganizada desde una perspectiva que no reconoció las diversidad de relaciones entre la generación de actividades económicas y el desarrollo de la vivienda y sus demandas de servicio. Debido a lo anterior, la hipótesis del trabajo aduce que las nuevas tendencias de movilidad, en particular la laboral, se observan alteradas en su intensidad y topologías, donde la geometría se ha tornado variable y difusa respecto de las situaciones anteriores, las transformaciones metropolitanas recientes aparecen significativas respecto de las condi- 
cionantes planteadas, por tanto agudizarían las tendencias observadas.

Así el proceso metropolitano es reciente, pero podemos aventurar que los desplazamientos no solo son más numerosos en general producto del proceso de expansión, sino que siguen destinos muy distintos al modelo periferia - centro, planteado en los comienzos de la década de 1980. Por lo tanto vale preguntarse: ¿dónde está y cómo se organiza espacialmente, el nuevo trabajo?, ¿cuáles son las nuevas topologías de las movilidades terciarias?, ¿existe promoción de nuevos modos de movilidad o solo es intensificación de los clásicos?

La metodología se centrará en dilucidar los elementos que están afectando los espacios de generación de viajes marcados por macromanzanas censales y aquellos espacios de tracción de viajes tomando en cuenta la separación fundamental entre empleo y trabajadores. Para ello, se espacializaron todas las nuevas patentes de servicios a nivel metropolitano y se trabajaron por aglomeraciones censales. Junto con ello, el levantamiento de las nuevas suburbanizaciones de la periferia de la metrópolis para contrarrestar espacios de generación de viajes y espacios atractores.

\section{La movilidad urbana en las lógicas metropolitanas}

\section{Sistemas de movilidad (PIG)}

La globalización económica y el crecimiento imparable del transporte motorizado son por consiguiente las dos caras de una misma moneda. Esta explosión generalizada de nueva demanda de transporte es preciso satisfacerla con grandes infraestructuras (viarias, portuarias, aeroportuarias...) que permitan un funcionamiento fluido de un modelo productivo que tiene una dimensión mundial. $Y$ al mismo tiempo esta creación de infraestructuras incentiva los procesos de globalización, urbanización y extensión de la movilidad motorizada (Estevan y Sanz, 1996).

Si bien parece claro que el crecimiento de las ciudades está relacionado con la capacidad de movimiento de personas, productos, información y energía, también cabe destacar que estas técnicas constituyen un sistema en sí. En la actualidad, las técnicas para el transporte de individuos, bienes e información (Graham \& Marvin, 2001) son completamente interdependiente, muy pocos movimientos son específicos, más bien, los recursos de infraestructura están en los tres dominios. El urbanista e investigador François Ascher lo Ilamó el sistema PIG (People, Information and Goods), enfatizando el vínculo que esto ha tenido en la historia de las ciudades. Él aduce que sin las técnicas de transporte y almacenamiento de mercancías, personas, información y energía, la expansión no hubiera sido posible.

Las consideraciones de expansión asociadas a las dinámicas PIG promueven el crecimiento expansivo de las ciudades y con ello el proceso de metropolización. Esto, contrario a las creencias de los investigadores y urbanistas de finales del siglo XX como los escritos de Melvin Webber en las décadas de 1960 y 1970 donde proclamaban que el transporte moderno y las telecomunicaciones iban a reducir la fricción del espacio y anticipaban la dispersión y desaparición de ciudades. En realidad el fortalecimiento es mutuo, el crecimiento de las ciudades y la concentración de ciertas actividades promueven el desarrollo del transporte y telecomunicaciones. Ascher (2004) explica que no debiera de ser de otro modo, ya que las tecnologías son en sí objetos sociales y no son independientes de la sociedad, sino que están enraizadas en ella, lo cual permite entender que sirven a los agentes que la dominan.

Las nuevas tecnologías de información y comunicación, junto con el rápido desarrollo del transporte privado han promovido la concentración urbana, y con ello, revitalizado las lógicas metropolitanas. Si bien ellas mismas presentan diferencias en su forma y dinámica, todas sustentan un modelo de extensión territorial que generan demandas de movilidad mayores y diferentes a las concentraciones que tradicionalmente se daban en espacios metropolitanos.

En este escenario la movilidad de los espacios urbanos o metropolitanos se ha multiplicado. Los viajes individuales son cada vez más numerosos, los motivos por desplazamiento cada vez más diversos y las 
distancias debido al crecimiento extensivo son mayores. El continuo crecimiento y la diversificación de la movilidad es tanto una consecuencia como un instrumento de estilos de vida contemporáneos, hasta el punto que "el derecho al trabajo, vivienda y educación, ahora implicarían el derecho a la movilidad" (Ascher, 2004).

\section{Aproximación sinérgica de la movilidad urbana}

En casi todas ciudades del mundo ya no hay duda alguna que el transporte y lo urbano se entrecruzan, se influyen y evolucionan juntos. Al parecer, atrás quedaron los análisis de principios de siglo XIX donde el paradigma de la causalidad estudiaba la infraestructura de transporte como un elemento que se inserta en el territorio, siendo este un causante o una consecuencia de los paradigmas urbanos. Donde el transporte era un factor explicativo o bien dependiente de los diferentes tipos de crecimiento de ciudad, y las diferencias en la tipología de tejidos se identificaban por las diversas infraestructuras de transporte.

Miralles-Guash (2002) plantea que el transporte urbano no es solo un elemento técnico introducido, sino que es, sí, una construcción social en la medida que el incremento de velocidad ha introducido nuevos conceptos de espacio y tiempo. En este escenario el modelo de movilidad que enfrentamos es el resultado de la necesidad de interacciones que el espacio metropolitano genera, pero, a la vez, las dinámicas metropolitanas son producidas o inducidas por el modelo de movilidad imperante. Entender la aproximación sinérgica o dialéctica (García Palomares, 2008: 5-24) del sistema nos permite abordar claves interpretativas no solo partiendo de la idea inicial que los cambios metropolitanos condicionan los desplazamientos, sino que también estos mismos cambios influyen en la movilidad y viceversa.

En este escenario trataremos de abordar la dialéctica asociada principalmente a tres evidencias importantes y de los cuales la data levantada nos permite dar luces sobre una movilidad urbana específica como es la laboral. Las tres evidencias primordiales que somos capaces de aventurar están asociadas al nuevo modelo productivo (postfordismo) y al cambio sociodemográfico (estructuras socioeconómicas) y cultural, y el acelerado proceso infraestructural (concesiones viales y arremetida pública estructurante).

\section{Movilidad y sociedad móvil, diferencias y desigualdades}

El estudio quiere plantear conceptualmente la diferencia paradigmática que tiene abordar el problema desde la movilidad y sus desplazamientos, en relación a los estudios basados netamente en el concepto de redes de transporte, flujos e infraestructura asociados a los primeros análisis de globalización o el camino que se debe recorrer para poder llegar a ello. La idea de comunidades móviles planteada por Ascher (Ascher, 2004: 352) y los metropolitans introducido por Soja (Soja, 2004), refiere a grupos de habitantes con características similares de movilidad pero sin poseer necesariamente una localización de vivienda en cercanía, eso lo demuestran fielmente Álvarez, Silva y Soto (2009) en la movilidad universitaria. Alumnos universitarios pertenecientes a un grupo etario común, caracterizados socialmente y con patrones centrífugos de localización de viviendas hacia la metrópolis. El punto es que son coaliciones o grupos de personas que tienen ciertas similitudes en el cómo se desplazan en la metrópolis o como aduce Soja (2004) en el cómo van reconociendo su condición social territorializada.

Si bien desde esta visión la movilidad emerge como algo distinto al transporte, reconociendo a este último como el componente material de ella, su definición presenta una polisemia (Gutiérrez, 2010) que va asociada a los distintos ángulos o dimensiones en el cómo se aborda: como un valor, un derecho o un capital social (Orfeil, 2004; Ascher, 2004; Kauffman, 2008). Esto permite entender en dónde se coloca la mirada, desde la "sociedad global" asociada a flujos, enlaces e infraestructura o desde un punto de vista más micro en la "sociedad hipermóvil", colocando el debate en la velocidad, los tiempos, la viabilidad de viaje (MirallesGuash, 2004), buscando quizás Ilegar a un análisis bottom-up. 


\section{Movilidad laboral: aquello que ha cambiado}

\section{Movilidad de masas y movilidad singular}

Asociado a esta última premisa de sociedad hipermóvil podemos plantear algunas caracterizaciones importantes desde una mirada metropolitana, enfrentando la movilidad laboral. Lo que ha cambiado es la diferenciación entre movilidad de masas y movilidad singular (García, 2009). La primera, ampliamente asociada a la etapa postfordista, donde los desplazamientos en su mayoría eran pendulares y concentrados en un espacio urbano distinguible, por ejemplo, los desplazamientos desde la periferia al centro metropolitano, o situados en áreas específicas de la ciudad, modelo que se repitió en la mayoría de las urbes latinoamericanas. Otro punto importante es el tiempo, los desplazamiento también se concentraban preferentemente en horas de entrada y salida del trabajo.

Los desplazamientos por trabajo o estudio (la movilidad habitual), dominante en la áreas urbanas tradicionales y con flujos claramente

Figura $\mathrm{N}^{\circ} 1$

Principales conexiones laborales diarias en el Gran Valparaíso

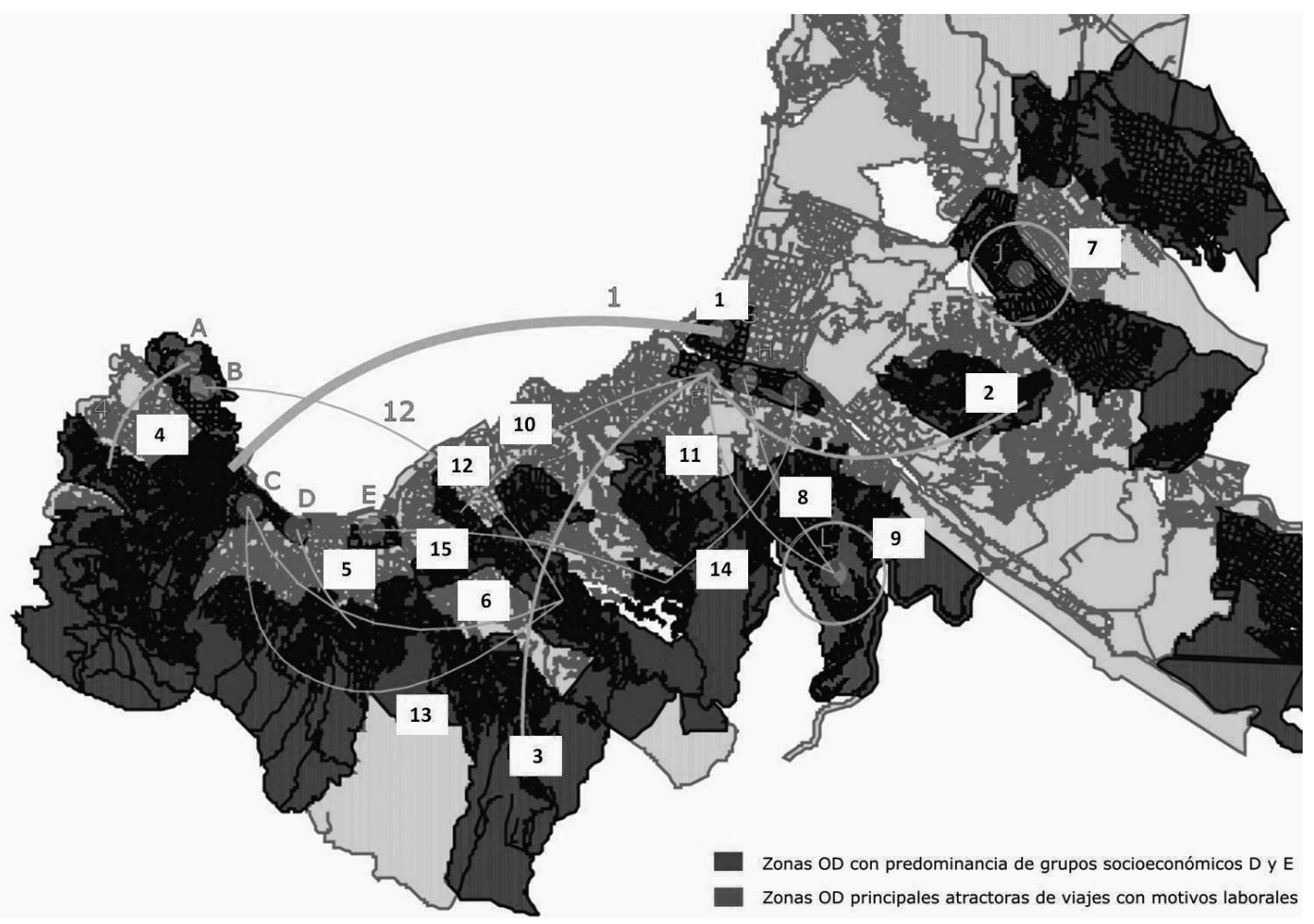

\begin{tabular}{|c|l|c|l|l|l|}
\hline 1. & Desde OD 11 a OD $91 / 1.562$ viajes & 6. & Desde OD 57 a OD $27 / 375$ viajes & 11. & Desde OD 68 a OD $88 / 320$ viajes \\
\hline 2. & Desde OD 107 a OD $88 / 636$ viajes & 7. & Desde OD 114 a OD $114 / 347$ viajes & 12. & Desde OD 57 a OD $8 / 319$ viajes \\
\hline 3. & Desde OD 50 a OD $88 / 604$ viajes & 8. & Desde OD 68 a OD $87 / 328$ viajes & 13. & Desde OD 57 a OD $28 / 319$ viajes \\
\hline 4. & Desde OD 4 a OD $1 / 559$ viajes & 9. & Desde OD 68 a OD $68 / 327$ viajes & 14. & Desde OD 61 a OD $86 / 313$ viajes \\
\hline 5. & Desde OD 39 a OD $28 / 401$ viajes & 10. & Desde OD 52 a OD $88 / 325$ viajes & 15. & Desde OD 61 a OD $36 / 308$ viajes \\
\hline
\end{tabular}

Fuente: Elaboración Jorge León en base a Encuestas de Origen y Destino, 2000. SECTRA-Norte). OD (Origen/ Destino). 
concentrados en su dirección (periferia-centro), son sustituidos por desplazamientos cuyos motivos son igualmente nuevos, pero en relaciones diversas (periferia-periferia e incluso centro-periferia) y acompañados por todo un conjunto de motivos de interés social, ampliamente diversificados (las nuevas formas de comprar), o siendo un motivo ya existente, que cobra una importancia en la actualidad muy Ilamativa (ocio). El desarrollo de los medios de transporte y las comunicaciones alarga las distancias cotidianas posibles, se multiplican las posibilidades de elección en una gran parte de la población en todos los aspectos de la vida urbana cotidiana (Ascher, 2004).

Los estudios de movilidad en los espacios han empezado a asumir que el tiempo utilizado en los desplazamientos no se reduce, a pesar de las mejoras que se producen en tecnología asociada a los medios de transporte. Los incrementos de velocidad en los desplazamientos $y$, por tanto, las reducciones de los tiempos de viajes son compensados con nuevos viajes y con mayores distancias.

La gente suele vivir en un lugar, trabajar en un segundo y situar su recreación en un tercero. Esto permite aventurar que no solo han aumentado los desplazamientos en cantidad, sino también los espacios desde donde se originan los viajes como aquellos que son "atractores", sobre todo en la periferia de nuestras ciudades. Más que un modelo centrípeto, es una nube de flujos lo que vemos hoy en día y se ve claramente reflejado en la Figura $\mathrm{N}^{\circ} 1$ del Valparaíso metropolitano, donde no se ven desplazamientos únicos, sino una nube de ellos.

Si bien el levantamiento está solo referido a lo que Edward Soja Ilama los "tránsitos dependientes", la clase social trabajadora de nivel socioeconómico más bajo, en Chile son el grupo "D y E" y que mayoritariamente solo usa el transporte público como medio de locomoción, son ellos el porcentaje mayoritario

Cuadro $\mathrm{N}^{\circ} 1$

Número de viajes por ocupación

\begin{tabular}{|c|c|c|}
\hline Ocupación & Viajes & Porcentaje \\
\hline Empresario (con trabajadores a su cargo) & 1.207 & 0,37 \\
\hline Profesionales independientes & 3.424 & 1,04 \\
\hline Profesional asalariado con jornada completa & 27.837 & 8,46 \\
\hline Profesional asalariado con jornada parcial & 4.072 & 1,24 \\
\hline Empleado no profesional (excepto FF.AA.) & 63.763 & 19,38 \\
\hline Miembro de FF.AA. y de Orden & 8.471 & 2,57 \\
\hline Trabajador independiente. No profesional & 32.715 & 9,94 \\
\hline Obrero no temporero & 36.521 & 11,1 \\
\hline Obrero temporero & 6.706 & 2,04 \\
\hline Dueña de casa & 20.843 & 6,33 \\
\hline Personal de servicio doméstico & 6.399 & 1,94 \\
\hline Estudiante & 103.809 & 31,55 \\
\hline Jubilado activo & 1.265 & 0,38 \\
\hline Jubilado inactivo (pensionado o montepiado) & 5.961 & 1,81 \\
\hline Cesante o busca trabajo por $1^{\mathrm{a}}$ vez & 4.451 & 1,35 \\
\hline Otro inactivo & 1.587 & 0,48 \\
\hline Total & 329.031 & \\
\hline
\end{tabular}

Periodo: Resto del día 0:00-7:15 / 9:15-9:30 / 12:45-13:00 / 14:15-14:30 / 17:45-18:00 / 19:30-24:00 Fuente: Elaboración propia en base a Encuestas de Origen y Destino, 2000. SECTRA-Norte). OD (Origen/Destino). 
de población al interior de lo metropolitano, con un $42,7 \%$, con 101.487 hogares en esa condición.

Junto con esto la flexibilidad laboral ha propiciado que los horarios de trabajo dejen de ser rígidos e únicos, por lo tanto también los tiempos de desplazamiento se diversifican y la data lo deja en evidencia. Este grupo mayoritariamente son empleados no profesionales que sustentan casi el $19,4 \%$ de los viajes y son el segundo grupo después de los estudiantes que tienen más viajes en la metrópolis. Es este grupo el que no tiene posibilidades de elegir lugares de residencia, por lo tanto están claramente alejados del lugar de trabajo y de los centros de servicios. Pero no solo ello ha ocasionado la diversidad en los motivos de viaje, sino que el aumento en las nuevas pautas de consumo ha alimentado un desplazamiento por ocio y servicios muy de la mano con la globalización cultural. De esta manera, cada uno de los habitantes de la ciudad tendríamos un propio modelo particular de movilidad (Cuadro $\mathrm{N}^{\circ} 1$ ).

\section{Factores detonantes de actual movilidad laboral en el Valparaíso metropolitano}

\author{
Transformación productiva \\ (terciarización de los servicios, \\ sociedades servindustriales)
}

En particular, el desarrollo urbano metropolitano de Valparaíso comienza su desarroIlo con el trazado ferroviario entre Valparaíso y Viña del Mar, seguido de las estaciones de Quilpué y Villa Alemana, por tanto el accionador histórico corresponde al ferrocarril, expresión fundamental de la revolución Industrial.

El proceso industrial es temprano y marginal en Valparaíso. Producto de sus condiciones morfológicas, Viña del Mar desarrolló sus ventajas de suburbio para articular la fase productiva inicial, de este modo sistemáticamente la ciudad comienza un desarrollo periférico obligando a extender el dominio de la movilidad industrial inicial, hacia la extensión del dominio metropolitano posible.
Las transformaciones productivas se han deslocalizado, un ajuste y redistribución territorial en distancia de alcances cada vez más metropolitanos, esta explicación es la más cercana a lo que denominamos postfordismo (García, 2008: 22).

La transformación productiva en el Gran Valparaíso comenzó con la pérdida progresiva del rol asignado a la producción hacia los puertos, culminando con la crisis de 1983, concuerda esto con el sostenido crecimiento de la movilidad dentro del área metropolitana, para el periodo censal 82-02, la movilidad se incrementó hasta alcanzar el $22 \%$ el porcentaje de población que se desplaza por razones de estudio y trabajo, el desaparecimiento de las industrias poco competitivas en el nuevo escenario, Fábrica y Refinerías de Azúcar de Viña del Mar (CRAV) y algunas textiles desaparecidas definitivamente a fines de 1980, se relocalizan para lograr más eficiencia dentro del nuevo escenario territorial creado, industrias de confites hacia Santiago (Hucke y Costa), Tabacalera (Compañía Chilena de Tabacos) hacia Casablanca.

Lejos de manifestarse en crisis de la ciudad con la que se asoció inicialmente esta descentralización, desde una perspectiva metropolitana, se ha reforzado el papel de las partes como centros de actividades terciarias. Asistimos a una redistribución territorial de centros industriales que sobreviven al proceso, la desaparición de aquellas poco funcionales a la nueva economía, y el surgimiento de nuevas actividades fuertemente dinamizadas por el contexto, por ejemplo, el desarrollo inmobiliario turístico que en el litoral de la Región de Valparaíso hoy concentra más del 65,5\% de las inversiones en el rubro, esta también la "agricultura urbana" del valle del Aconcagua y Casablanca, dependientes de los servicios (tecnologías, conocimiento, expertizaje, masa laboral expresada en el temporero) que la ciudad dispone y que acompaña la revitalización de las economías metropolitanas y su principal manifestación territorial es la movilidad y la ciudad es la maquinaria de servicio para las dinámicas periféricas y suburbanas en donde hoy tiene su mayor expresión las transformaciones y la visibilidad de lo "modernizado". 


\section{El modelo de desarrollo periférico y difuso}

Podemos señalar que en el Gran Valparaíso la dinámica urbana tiene una fuerte componente en las periferias de la misma, lo que asegura una articulación robustecida en las periferias, más dinámicas, flexibles y accesibles.

Fuera de los límites urbanos y asociado a las redes estructurantes interregionales se ha presentado un fuerte proceso de periurbanización, los antecedentes señalan que entre 1987 y 2007 (años de crecimiento sostenido) se registraron en la Región de Valparaíso 1.121 cambios de uso de suelo, incorporando 39.435,5 hectáreas.

El periodo tratado se entiende de fuerte dinamismo económico inmobiliario, reactivación promovida postrecesión, el ajuste normalmente se desencadena a través de la especulación inmobiliaria o reajuste espacial, principal promotor y dinamizador de las periferias de las ciudades, las regiones y los centros generales. A esto se agrega un elemento de fuerte cualificación territorial, la puesta en vigencia de la "Ley de Concesiones" de obras públicas para las infraestructuras viales.

El reajuste o expansión territorial requiere una serie de articulaciones para reubicar los excedentes, espacialmente siempre la periferia genera márgenes más interesantes, por otro lado la instrumentalización vigente obliga a un número significativo de procedimientos, manifestándose al final del camino en una serie de resoluciones favorables al cambio de uso, donde la cualidad "silvoagropecuario" significa minusvalía y postergación territorial, su inserción al expediente urbano, al menos asegura el menor valor de suelo urbano, normalmente 2,5 veces respecto del mayor valor silvoagropecuario, para la región estudiada es el suelo inundable.

Si se toman las $39.435,5$ hectáreas comprometidas en cambios de uso de suelo y la acreción natural del Gran Valparaíso para el mismo periodo (sin considerar la densificación), la superficie comprometida como cambio de uso duplica esta superficie. Claramente el espíritu de la ley se vulnera en favor del especulador, y a su vez subutiliza el suelo, toda vez que la ocupación del suelo es objeto de cambio de uso, se utiliza parcialmente a través de la asignación de condiciones de urbanización, promovidas por la competencia territorial local.

Todas estas dinámicas fuera del límite urbano pero claramente de procedencia urbana generan trabajo de distancias y obligan a los desplazamientos desde las residencias al interior de las ciudades hacia las "urbanizaciones", esta nueva reconfiguración hace más dinámicas las periferias históricamente postergadas y marginales en sus desarrollos, hoy están en el centro de las nuevas estrategias inmobiliarias, fundamentalmente turísticas, por tanto más dependiente de las poblaciones de servicios, incrementando los desplazamientos, ahora también hacia las periferias, un componente complementario pero que genera transformaciones sobre las dinámicas regulares del área.

\section{Transformaciones en la distribución socioeconómica}

La distribución de la estructura socioeconómica ${ }^{4}$ se ha recompuesto, y el resultado es una composición bastante desigual entre las comunas que configuran el área metropolitana de Valparaíso.

En el análisis, si utilizamos la línea de tendencia podemos señalar que se encuentran relativamente en equilibrio, Concón, Viña del Mar y Quilpué, aunque distorsionada en la base del grupo $A B C 1$, tendiente a la alza y con menos participación del grupo C3 (Figura $\mathrm{N}^{\circ} 2$ ).

El caso de Valparaíso ciudad, esta presenta la curva más irregular respecto del promedio y el equilibrio deseado, ausencia casi total del grupo $A B C 1, y$ aumento sustantivo en los grupos $\mathrm{D}$ y $\mathrm{E}$, el caso de Villa Alemana aglutina un grueso de clase media C3, por

\footnotetext{
4 El dato de la estructura socieconómica se obtiene a partir de los datos censales donde se genera una matriz de doble entrada que cruza la escolaridad promedio de los integrantes de un hogar y los bienes que declaran disponer, modelo adaptado por Adimark, 2004.
} 
sobre el promedio, y se mantiene estable respecto del resto.

Relacionando estructura socioeconómica y masas laborales, deberíamos sugerir que gran parte de los volúmenes desplazados se mantienen en sus residencias, la movilidad residencial opera con los segmentos de clase media (C3) y (ABC1), normalmente hacia las periferias (Villa Alemana y Curauma por ejemplo), confinando los sectores modestos respecto de sus desplazamientos laborales hacia áreas cada vez más distantes pero fuertemente demandadora de bienes y servicios de la ciudad. El desplazamiento es hacia los contextos pericentrales del Gran Valparaíso, tal como se señala en la explicación del imperio de servicio o terciarización urbana (Cuadro $\mathrm{N}^{\circ} 2$ ).

\section{El acelerado proceso infraestructural} (concesiones viales y arremetida pública estructurante)

El espacio de la Macrorregión central (en adelante MCZ), contexto del área metropolitana de Valparaíso, concentra hoy las mayores inversiones privadas concesionadas, si consideramos solo las cuatro obras concesionadas que unen la Región Metropolitana y la

Figura $\mathrm{N}^{\circ} 2$

Valores absolutos (cantidad de hogares)

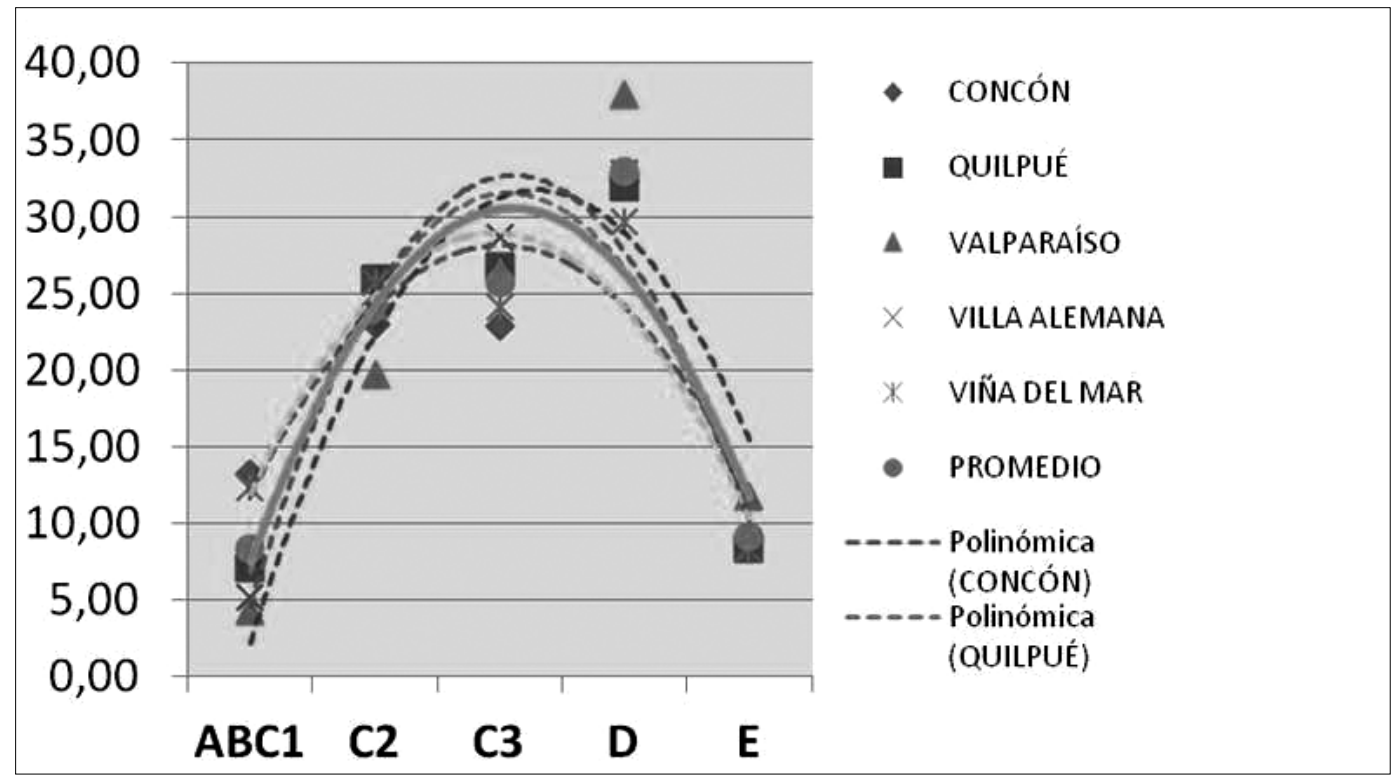

Fuente: Elaboración propia en base a Censo 2002.

Cuadro $\mathrm{N}^{\circ} 2$

Distribución socioeconómica por comunas del Gran Valparaíso

\begin{tabular}{|l|r|r|r|r|r|r|}
\hline Comuna & ABC1 & \multicolumn{1}{|c|}{ C2 } & \multicolumn{1}{c|}{ C3 } & D & E & \multicolumn{1}{c|}{ N $^{\text {Hogares }}$} \\
\hline Concón & 1.192 & 2.081 & 2.067 & 2.920 & 800 & 9.060 \\
Quilpué & 2.659 & 9.730 & 10.036 & 11.965 & 3.139 & 37.529 \\
Valparaíso & 3.328 & 15.515 & 20.772 & 29.831 & 9.196 & 78.642 \\
Villa Alemana & 1.416 & 6.883 & 7.865 & 9.040 & 2.281 & 27.485 \\
Viña del Mar & 10.435 & 21.880 & 20.500 & 25.233 & 7.082 & 85.130 \\
Gran Valparaíso & 19.030 & 56.089 & 61.240 & 78.989 & 22.498 & 237.846 \\
\hline
\end{tabular}

Fuente: Elaboración propia en base a Censo 2002. 
Región de Valparaíso Costa (Ruta 68, Ruta 78, Troncal Sur, Litoral Central) la inversión en los últimos cinco años asciende a 795 millones de dólares, esto equivale a nueve veces la inversión pública de obras públicas en toda la Región de Valparaíso en los mismos cinco años.

Si consideramos la inversión privada distribuida a lo largo de los 10 años, periodo en el cual se ha desarrollado la inversión vía concesión en los proyectos señalados, tenemos que el $89 \%$ corresponde a inversión privada y el $11 \%$ a inversión pública.

Hasta el año 2010 existían trece proyectos viales que han sido adjudicados al sector privado, los cuales involucran una inversión cercana a los US\$ 3.000 millones, muy similar a las inversiones públicas en todo tipo de infraestructura durante la última década.

La red vial interurbana de la MCZ está formada por $10.357 \mathrm{~km}$ y muestra un desarroIlo relativo mayor que al del resto de Chile. En efecto, el $27,7 \%$ de estos caminos tiene carpeta de rodado, ya sea asfáltica o de hormigón, mientras que el promedio nacional es de solo 13,9\%. La Región Metropolitana es a su vez la que mayor proporción de caminos pavimentados tiene, con un $51,5 \%$.

Por otro lado, a escala regional, el "Plan Urbano Regional" de la Región de Valparaíso, en su propuesta de desarrollo de orbitales intrarregionales, se orienta positivamente a integrar, por una parte, el sistema urbano de borde costero, y por otra, plantea orbitales en las "espaldas", estableciendo condiciones para desconcentrar el crecimiento urbano de los sistemas saturados de Valparaíso, hacia corredores de transporte que plantean vías alternativas de interconexión, todo esto acrecienta las ventajas de la proximidad. Estas ventajas generadas han producido entre el año 1997 y el año 2007 (10 años del proceso de concesiones), cambios significativos en la repartición modal de los flujos en el transporte de pasajeros, aunque no así en el de cargas. La proyección tendencial apunta a un fuerte aumento en el empleo de los automóviles, que pasa de un $21 \%$ de los viajes diarios en 1997 al 36\% en el año 2007.
El flujo diario de pasajeros sube de 13,7 a 22,2 millones de viajes, mientras que el número de pasajeros $/ \mathrm{km}$ va de 246 millones a 573, representando un aumento del $133 \%$.

\section{La distribución de las redes de transporte y las dinámicas de movilidad en el área metropolitana de Valparaíso}

Se utiliza la matriz 2009 de información de viajes laborales dentro del Gran Valparaíso (comunas de Valparaíso, Viña del Mar, Villa Alemana, Quilpué y Concón), desarrollada por la SECTRA utilizando el modelo proyectivo ESTRAVAL a partir de los datos de la encuesta de origen-destino del año 2001.

Lo que podemos observar en términos generales, que para cada zona existen sistemas de ajustes y reajustes para generar equilibrios bastantes balanceados, o sea, en general podemos señalar que las unidades territoriales que más se desabastecen de habitantes, también son las que más recepcionan, generando un equilibrio que rompe la inercia histórica de carácter pendular entre las ciudades denominadas del interior y la costa (Quilpué y Villa Alemana respecto de Valparaíso y Viña del Mar).

Para identificar estos sistemas funcionales y las transformaciones de estas nuevas centralidades o también Ilamada "nodalización urbana", existe una técnica designada como el eslabón principal, la cual, a partir de una matriz de flujos de interacción entre zonas del área, permite identificar las relaciones de dependencia entre ellas y detectar los sistemas espaciales (Silva, 2008) (Figura $N^{\circ} 3$ ).

Es una forma de descubrir las centralidades mediante indicadores de movilidad urbana. En nuestro caso se realizó el análisis de eslabón principal tomando como base las matrices de Origen-Destino de Viajes en el Gran Valparaíso, realizado por SECTRA el año 2000. Los resultados que muestra el análisis, realizado para todos los viajes en todos los modos de transporte, adicionalmente se analizó la situación de "hora punta" en la mañana, en modo locomoción colectiva, como una forma de establecer la situación 
bajo modalidad de transporte masivo de pasajeros y la estructura funcional de la ciudad cambia.

El resultado del primer análisis que considera todos los viajes en un día, en todos los modos de transporte sin discriminar el motivo del viaje. Se observan tres nodos importantes en el sistema, Valparaíso, Viña y Quilpué. Los dos primeros constituyen sistemas cerrados y servidores de sus propias periferias que rodean los distritos de negocios y servicios en el plan de cada ciudad.

El considerar todos los viajes en todos los modos de transporte, estadísticamente el efecto es suavizar los fenómenos y se tiende a homogeneizar los distintos dominios urbanos de interacción de los habitantes. Sin embargo, muestra una situación promedio y estable en el largo plazo, con esto, además se evidencian las relaciones funcionales de los sistemas urbanos más estables en el tiempo.

En cambio podemos establecer una visión más coyuntural y cotidiana de la movilidad, muestreando un período punta y en modo de transporte diario masivo de pasajeros. En segundo análisis, consideramos la movilidad en un período punta como el de la mañana y en el modo de locomoción colectiva, y veremos la configuración funcional que adquiere el espacio urbano en este escenario.

Prácticamente todas las áreas se eslabonan con el plan de Valparaíso, reduciéndose la red nodal a solo dos núcleos, los centros de Valparaíso y Viña del Mar. Se observa además que el único eslabonamiento de ida y vuelta se produce entre los centros de Viña y Valparaíso. Esto demuestra la gran interdependencia que han adquirido con el tiempo y es una tendencia que no desaparece.

En esta coyuntura, aparecen dependientes funcionalmente de Valparaíso, incluso las ciudades de Quilpué y Villa Alemana, que en esquema anterior surgieron como un subsistema independiente.

Se plantea un desafío para la gestión urbana de la intercomunal, pues es un sistema urbano que evidencia una habitación en sus periferias muy dependiente de los centros ur-

Figura $\mathrm{N}^{\circ} 3$

Distribución sectores sociales de mayor movilidad

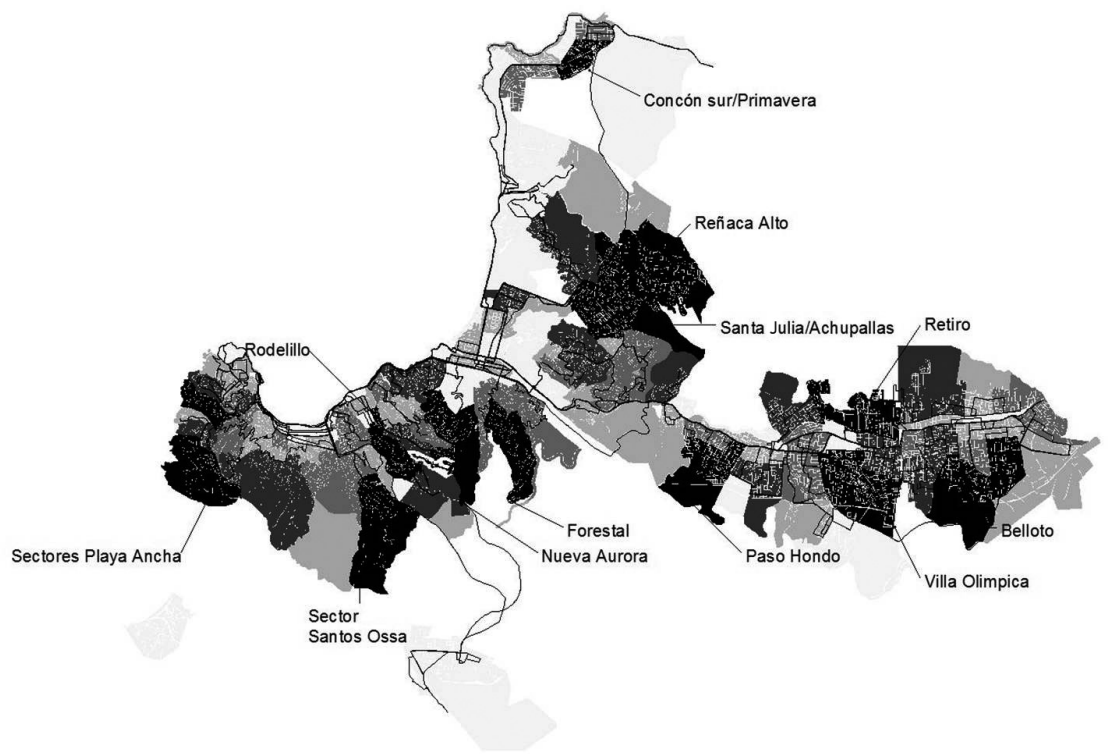

Fuente: Elaboración propia en base a Encuesta Origen y Destino, 2002. SECTRA Norte. 
Figura $\mathrm{N}^{\circ} 4$

Distribución de Zonas OD (Origen y Destino) Atractoras.

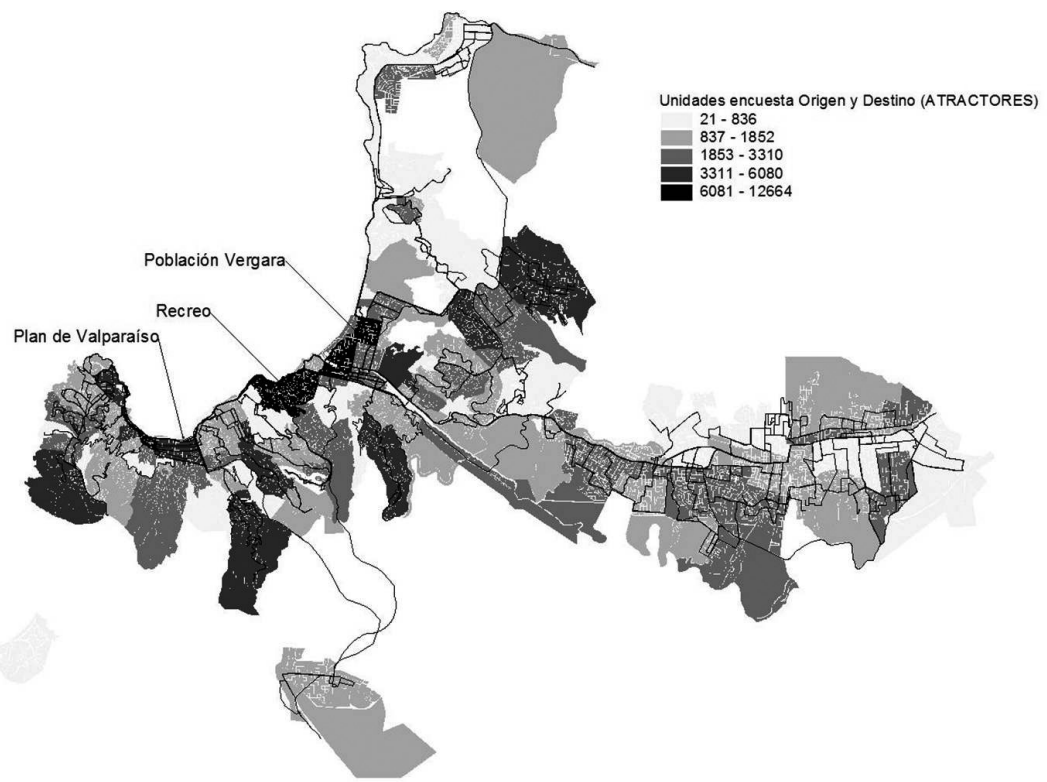

Fuente: Elaboración propia en base a Encuesta Origen y Destino, 2002. SECTRA Norte.

Figura $N^{\circ} 5$

Distribución de Zonas OD (Origen y Destino) Expulsoras.

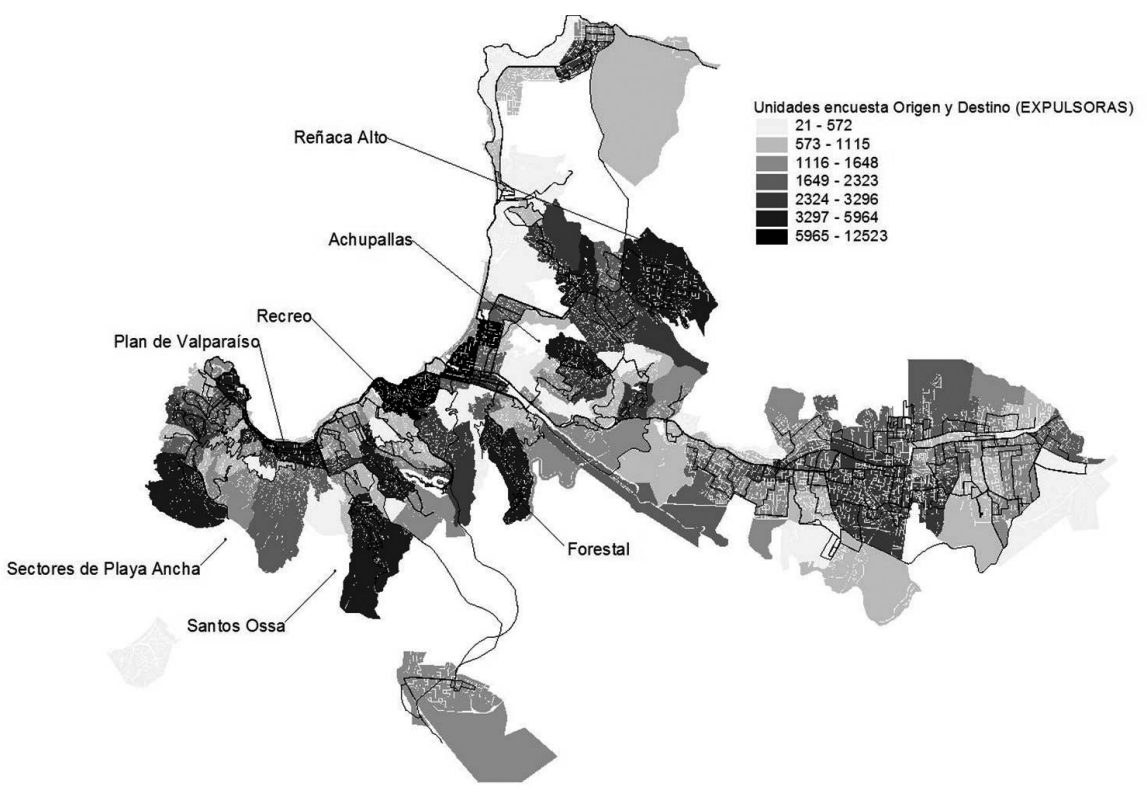

Fuente: Elaboración propia en base a Encuesta Origen y Destino, 2002. SECTRA Norte. 
banos ubicados en el plan, y sujetas a restricciones de una topografía bastante accidentada que no hace fácil organizar el crecimiento urbano ni establecer una red vial que dé alternativas de movilidad a los desplazamientos urbanos diarios de sus habitantes.

Los desarrollos de periferia, como criterio de calidad, no deben presionar el centro urbano principal, y este criterio de diseño se refiere a un crecimiento de descentralización concentrada, es decir, en conjuntos residenciales equipados que impidan viajes a los centros históricos (Figura $N^{\circ} 4$ ).

Se puede observar en la Figura $\mathrm{N}^{\circ} 4$ que las unidades fuertemente "Expulsoras" son las periféricas como los sectores de Playa Ancha, Santos Ossa, Forestal Santa Julia y Reñaca Alto. Pero también están las zonas que entendemos generan atracción por concentración de actividades como el Plan de Valparaíso, Plan de Viña del Mar, en donde se produciría un fuerte recambio entre residentes y población laboral flotante, de carácter diario.

La Figura $N^{\circ} 5$ complementa el análisis indicando dónde se encuentran las principales zonas "Receptoras".

Podemos observar que no se presentan grandes transformaciones y la tendencia es a equilibrarse, la regla no se cumple con algunas áreas o zonas periféricas de ValparaísoQuilpué y Villa Alemana. Algunos sectores altos (cerros) de Valparaíso en Playa Ancha y Almendral presentan diferencias entre la emisión y recepción de los destinos por viales laborales, lo mismo sucede con los sectores populosos de Quilpué y Villa Alemana, tanto para el área norte y sur, respectivamente. Para los sectores de la Meseta de Viña del Mar, el escenario cambia para Santa Julia. AchupaIlas, Glorias Navales y Reñaca Alto, manteniéndose equilibrado en los flujos los sectores del sur de Viña del Mar, Reñaca y Concón.

Las dinámicas laborales y los desplazamientos

Para el estudio de las dinámicas económicas locales se procedió a la espacialización de las patentes municipales, corresponde entonces a la distribución formal de las actividades económicas de las ciudades que configuran el Gran Valparaíso. La concentración en términos relativos se localiza en Viña del Mar, con el $47 \%$ de las actividades. Por el contrario, la menor concentración se encuentra en Concón con solo el 3\% de las patentes (Cuadro $\mathrm{N}^{\circ} 3$ ).

Cuadro $\mathrm{N}^{\circ} 3$

Patentes municipales en el Gran Valparaíso

\begin{tabular}{|l|r|r|}
\hline Comunas & Total de patentes & Porcentaje \\
\hline Valparaíso & 8.076 & 26 \\
\hline Viña del mar & 14.666 & 47 \\
\hline Concón & 989 & 3 \\
\hline Quilpué & 5.495 & 17 \\
\hline Villa Alemana & 2.233 & 7 \\
\hline Gran Valparaíso & 31.459 & 100 \\
\hline
\end{tabular}

Fuente: Elaboración propia.

Cuadro $\mathrm{N}^{\circ} 4$

Número de patentes municipales y total de hogares

\begin{tabular}{|l|r|r|r|}
\hline \multicolumn{1}{|c|}{ Comunas } & \multicolumn{1}{c|}{ Total de patentes } & Total hogares & Tasa: actividades por hogar \\
\hline Viña del Mar & 14.666 & 85.130 & 5,8 \\
\hline Quilpué & 5.495 & 37.529 & 6,8 \\
\hline Concón & 989 & 9.060 & 9,2 \\
\hline Valparaíso & 8.076 & 78.642 & 9,7 \\
\hline Villa Alemana & 2.233 & 27.485 & 12,3 \\
\hline Gran Valparaíso & 31.459 & 237.846 & \\
\hline
\end{tabular}

Fuente: Elaboración propia. 
Ahora, si utilizamos la referencia de la cantidad de hogares en cada una de las ciudades, tenemos que las dos mayores concentraciones se encuentran en Viña del Mar y Quilpué, situación encontrada con la regularidad histórica que lideró Valparaíso, hoy relegada al cuarto lugar relativo, en dinámicas económicas, quizás su dimensión administrativa, no estudiada aquí, sea una de las que aún sostiene y lidera metropolitanamente, pero en cuanto a actividades económicas la centralidad se ha desplazado, por consiguiente también sus desplazamientos (Cuadro $\mathrm{N}^{\circ} 4$ ).

El patrón regular se ha reconfigurado, dispersándose y reconcentrándose donde la centralidad geográfica de Viña del Mar tiene favorables condiciones para capitalizar el nuevo escenario.

Estudiadas las actividades respecto de su topología se pueden reconocer unidades te- rritoriales bastante claras y de una accesibilidad relativa fuertemente vinculada a la redes estructurantes principales, las cuales generan la acción diseminadora sobre el total del contexto periurbano del Área Metropolitana de Valparaíso (Figura $N^{\circ} 6$ ).

Considerando las densidades de patentes, (concentraciones topológicas, de acuerdo a patrones de distribución en torno a la calle menores a 25 metros), podemos señalar que las mayores concentraciones las tiene Viña del Mar en sus tramos históricos del centro histórico y de las extensiones urbanas de la misma en torno a la calle Quillota y Libertad hasta 15 Norte, esta concentración se explica por una relación de centralidad geográfica pivotante, en donde las relaciones de orden infraestructural la fortalecen y organizan, por otro lado la segunda unidad es la concentración histórica del "Plan de Valparaíso", entre el sector Aduana o Puerto hasta el Almendral, cubriendo el sector llano de la ciudad primada.

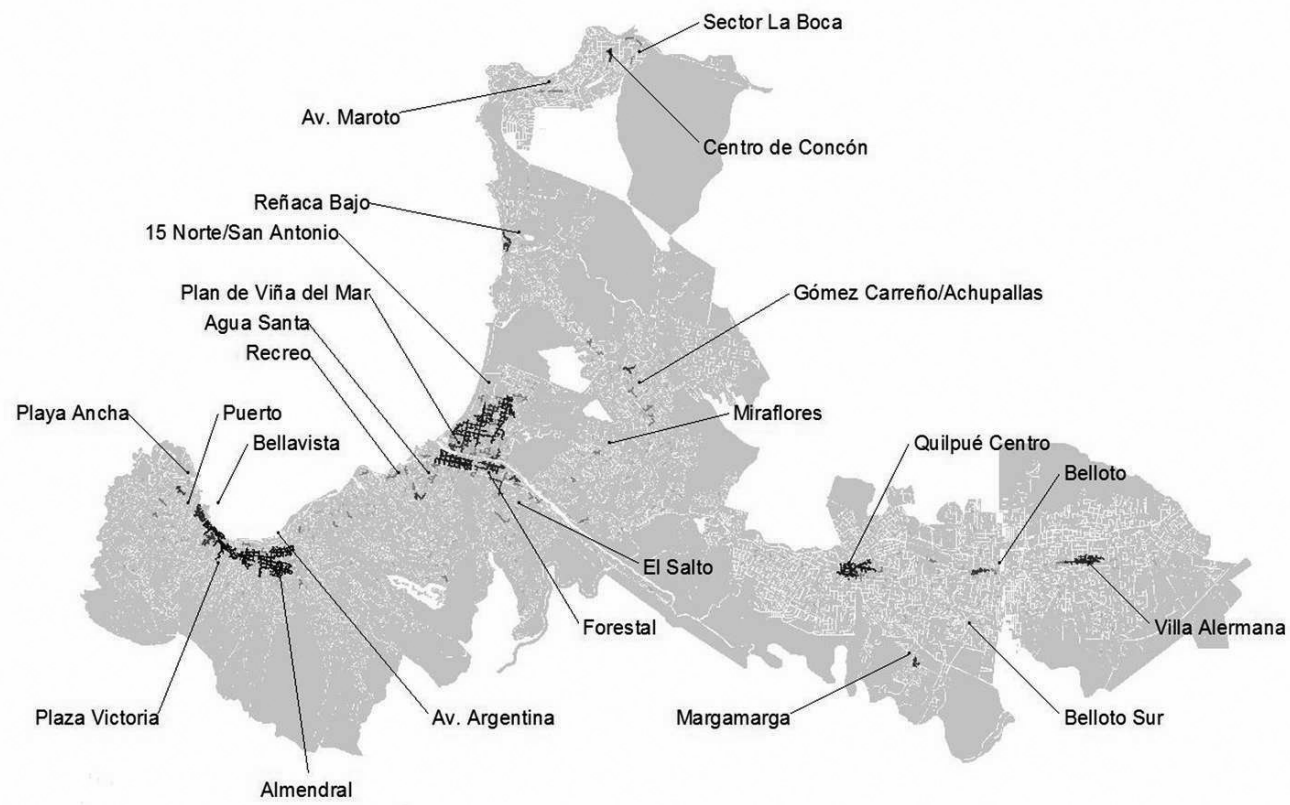


El resto de la organización se encuentra distribuido en torno a las redes principales y los centros históricos de Concón, Quilpué y Villa Alemana, el caso del sector del Belloto (dentro de Quilpué) pero hacia el límite de Villa Alemana, presenta una centralidad de actividad significativa y reciente, de todas maneras las actividades al interior de las comunas se encuentran fuertemente alineadas con la red estructurante metropolitana histórica más relevante; el "camino troncal".

Por otro lado, si a estas concentraciones le agregamos los servicios de transporte colectivo masivos como los microbuses, se obtiene la Figura $N^{\circ} 7$.

Para los tres recorridos más extensos del sistema de transporte colectivo dentro del Gran Valparaíso, estos necesariamente inter- ceptan las cinco principales concentraciones metropolitanas, superponiendo incluso sus recorridos, claramente aprovechando las ventajas desarrolladas en estos lugares, el transporte público cataliza las dinámicas de centralidad favoreciéndolas y declarándoles un mayor nivel de accesibilidad.

\section{Conclusiones}

La estructura urbana metropolitana del Gran Valparaíso se recompone, en un afán histórico de crecimiento por acreción y fuertemente jerarquizado con una ciudad primada como Valparaíso, a un proceso más flexible en sus límites y periferias y a una centralidad geográfica funcional a los desplazamientos y a las redes infraestructurantes que la satisfacen y permiten un funcionamiento fluido de un modelo productivo.

Distribución sistemas de recorrido, transporte metropolitano de Valparaíso.

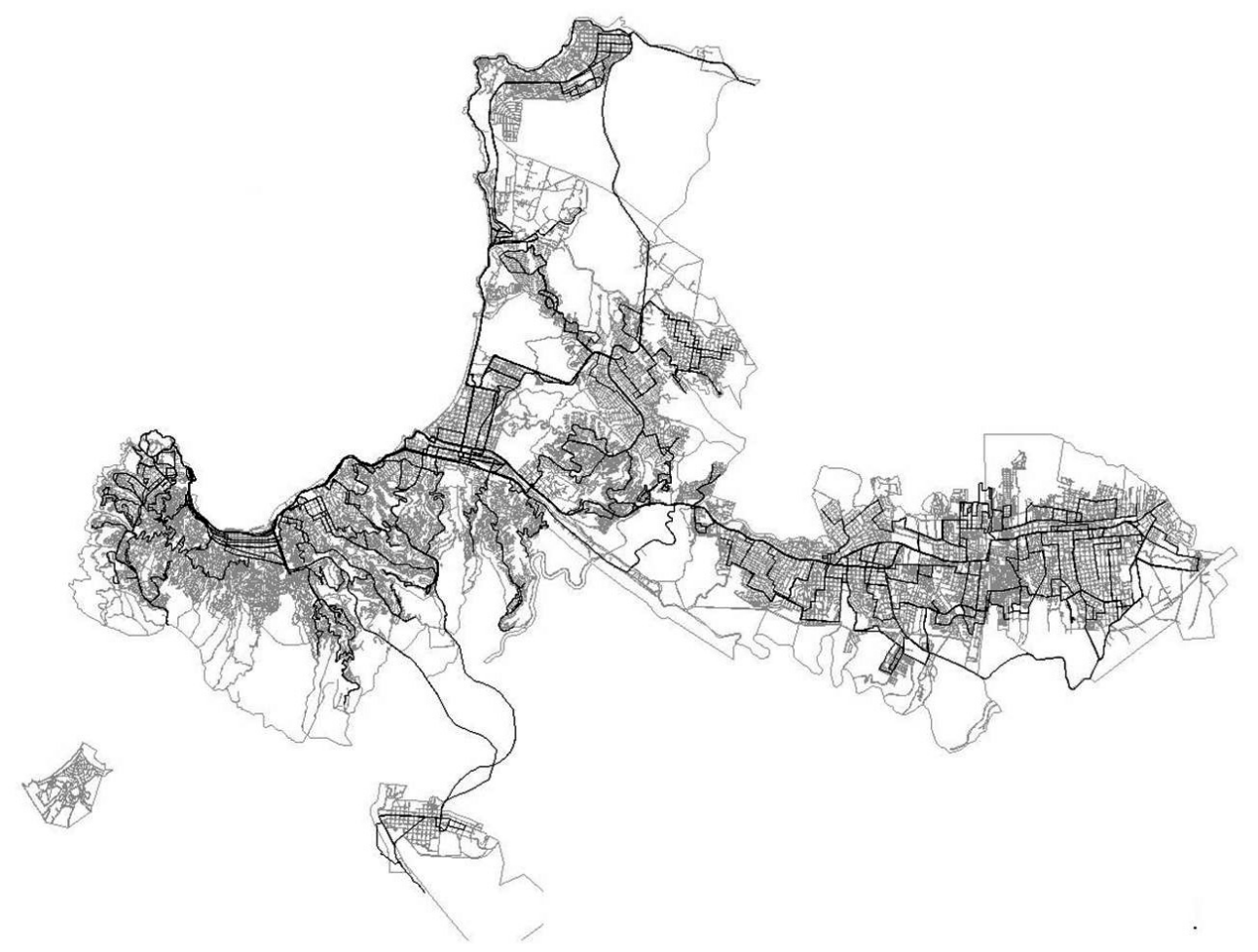

Fuente: Elaboración propia en base a Encuesta Origen y Destino, 2002. SECTRA Norte. 
Las nuevas accesibilidades construidas en los últimos años en el área metropolitana de Valparaíso y la macrorregión central, aumentan la movilidad de personas y proveedores hacia áreas periurbanas del conurbado.

Las sociedades se han tornado "hipomóviles", desde una perspectiva metropolitana estas se complejizan y tienden a singularizarse, en el sentido de que históricamente las condiciones de movilidad en el Gran Valparaíso se entendían como pendulares entre las así llamadas "ciudades dormitorios" y el "centro" de las dinámicas económicas, distinguible por el Valparaíso histórico. Hoy vemos singularidades, como por ejemplo el desplazamiento desde el centro a las nuevas periferias, traslados entre periferias (de Viña del Mar a Concón o de Quilpué a Placilla).

Las transformaciones deberían repercutir en las escalas micro, donde las alteraciones en la velocidad, los tiempos y lo viable de los viajes, deberían tener consecuencias en las oportunidades de las personas. La accesibilidad no se universaliza o generaliza en el Gran Valparaíso, las excepcionales condiciones topográficas le colocan fricción al territorio y lo que aparentemente está "integrado" como estructura urbana puede no estar "conectado".

Promover la movilidad con una postura de incrementar la conectividad es también a futuro la oportunidad del habitante de "llegar a tiempo" para mejorar su condición o medrar a través de la generación de accesibilidad, el territorio se comprime solo para algunos sectores del Gran Valparaíso, para otros el "retraso" como referente del rezago utilizado hasta ahora para la caracterización de estos sectores.

El modelo de movilidad que enfrentamos es el resultado de la necesidad de interacciones que el espacio metropolitano genera, pero a la vez, las dinámicas metropolitanas son producidas o inducidas por el modelo de movilidad imperante.

Por otro lado las principales transformaciones dicen relación con la distribución de la estructura socioeconómica, se ha recompuesto, y el resultado es una composición bastante desigual entre las comunas que con- figuran el área metropolitana de Valparaíso, lo que redunda en una asimetría respecto de las masas laborales cada vez más segregadas y desintegradas.

Relacionando estructura socioeconómica y masas laborales, deberíamos sugerir que gran parte de los volúmenes desplazados se mantienen en sus residencias, la movilidad residencial opera con los segmentos de clase media $(\mathrm{C} 3)$ y $(\mathrm{ABC} 1)$, normalmente hacia las periferias (Villa Alemana y Curauma por ejemplo), confinando los sectores modestos respecto de sus desplazamientos laborales hacia áreas cada vez más dinámicas, y evidentemente estas están ubicadas en los contextos pericentrales del Gran Valparaíso.

Podemos señalar que en el Gran Valparaíso la dinámica urbana tiene una fuerte componente en las periferias de la misma. Fuera de los límites urbanos y asociado a las redes estructurantes interregionales se ha presentado un fuerte proceso de periurbanización, los antecedentes señalan que entre 1987 y 2007 (años de crecimiento sostenido) se registraron en la Región de Valparaíso 1.121 cambios de uso de suelo incorporando 39.435 hectáreas.

La vida de las personas en el Gran Valparaíso y el funcionamiento de las organizaciones son cada vez más independientes de las zonas urbanas, los límites físicos y administrativos, no importa cómo estos se definan, la identificación de cada unidad comunal con una ciudad homónima comienza a diluirse, creada la comuna de Concón, a esta no se le identifica como "ciudad", entendiendo la parte de un todo urbano metropolitano. Hoy la gente suele vivir en un lugar, trabajar en un segundo y situar su recreación en un tercero.

Más que un modelo centrípeto y pendular, lo que hoy se observa es una nube de flujos superpuestos, "diagonalizando y circunvalando" como principio de una nueva geometría del territorio.

\section{Referencias bibliográficas}

HERCE, M. Sobre la Movilidad en la Ciudad. Barcelona, España Editorial Reverte, 2009. 
ASCHER, F. Los nuevos principios del urbanismo. Madrid: Alianza Editorial. 2004.

ASCHER, F. Multi-Mobility, Multispeed Cities: a challenge for architects, town planners and politicians. In: FONT A.; LLOP, C.; BERNADÓ, J. \& COLEGIO DE ARQUITECTOS DE CATALUÑA (editors). The explosion of the City, Morphologies, Observations and Motions. Barcelona: Universal de las Culturas, 2004, p. 352-363.

BERTOLINI, L. Fostering Urbanity in a Mobile Society: Linking Concepts and Practices. Journal of Urban Design, 2006, Vol. $11, N^{\circ} 3$, p. 319-334.

CÁCERES, G. y SABATINI, F. Para entender la urbanización del litoral; el balneario en la conformación del Gran Valparaíso (siglos XIX y XX). $A R Q, 2003, N^{\circ}$ 55, p. 50-52.

CASTELLS, M. The Informational city: information technology, economic restructuring, and the urban-regional process. Oxford: Editorial Basil Blackwell, 1991.

ESTEVAN, A. y SANZ, A. Hacia la reconversión ecológica del transporte en España. Madrid: Libros La Catarata. 1996.

FONT, A. (editor). L'explosió de la ciutat. Morfologies, mirades i mocions. Barcelona: Universal de las Culturas, 2004.

GARCÍA, J.C. Incidencia en la movilidad de los principales factores de un modelo metropolitano cambiante. EURE, 2008, Vol. XXXIV, N 101, p. 5-24.

GARCíA, J.C. Los desplazamientos al trabajo en la comunidad de Madrid. Madrid: Ediciones GPS, 2008.

GUTIÉRREZ, A. Movilidad, transporte y acceso: una renovación aplicada al ordenamiento territorial. Scripta Nova. Revista electrónica de Geografía y Ciencias Sociales, 2010, Vol. XIV, № 331, Disponible en Internet: http://www.ub.es/geocrit/sn/sn-331/sn331-86.htm

HARVEY, D. The conditions of posmodernity: an enquiry into the origins of cultural change. Oxford: Editorial WileyBlackwell, 1991.

HERCE, M. Sobre la movilidad en la ciudad. Propuestas para recuperar un derecho ciudadano. Barcelona: Editorial Reverté, 2009.

HIDALGO, R. y BORSDORF, A. Puerto Abierto. ¿Ciudad Cerrada?, Transformaciones Socio-espaciales en la estructura urbana del Área Metropolitana de Valparaíso. Revista Geográfica de Valparaíso, 2005, № 36.

JIRÓN, P.; LANGE, C. y BERTRAND, M. Exclusión y desigualdad espacial: Retrato desde la movilidad cotidiana. Boletín del Instituto de la Vivienda, 2010, Vol. 25, № 68, p. 15-57.

MIRALLES-GUASCH, C. Ciudad y transporte. El binomio imperfecto. Barcelona: Editorial Ariel, 2002.

MONCLÚS, F.J y OYÓN, J.L. Transporte y crecimiento urbano en España, mediados s. XIX - finales s. XX. Estudios Territoriales, 1996, Vol. XXVII, No 107-108, p. 217-240.

SILVA, L. Diagnóstico económico de Viña del Mar. 2008 (Inédito).

SOJA, E. De la Ciutat a I'Urba. Revista Àmbits de Política i Societat, 2004, No 29, p. 62-75.

SOJA, E. Postmetropolis. Critical studies of cities and regions. Oxford: Editorial Blackwell, 2000.

URRY, J. Sociology beyond societies: mobilities for the twenty-first century. Londres: Editorial Routledge, 2000.

WEBBER, M. El orden en la diversidad: comunidad sin proximidad. En: Ciudades y espacio. El uso futuro del suelo urbano. Barcelona: Editorial Oikos-Tau, 1976, p. 19-27.

ZAHAVI, J. A new Urban Travel Model. Presentación en IFIP Working Conference on Global Modelling, 1980. Disponible en Internet: http://www.surveyarchive.org/zahavi. html 